\title{
TROMLYCA GÉNERO NUEVO EN LOS ANDES DE COLOMBIA (RUBIACEAE, PALICOUREEAE)
}

\author{
AtTila L. Borhidi \\ Instituto de Biología, Universidad de Pécs H-7624 Pécs, Ifjúság útja 6, Hungría \\ E-mail: borhidi@gamma.ttk.pte.hu
}

(Received 22 February, 2017; Accepted 15 March, 2017)

The new genus is based on Palicourea locellata C. M. Taylor described from the Colombian Andes and placed into the Nonatelia section reconsidered and strongly enlarged by $\mathrm{C}$. M. Taylor including 26 species into the originally monotypic section. According the new protologue of the section it is a highly varied and rather heterogeneous group in morphological point of view. Therefore the section Nonatelia C. M. Taylor is interpreted here in a reduced sense including not more than 5 species. The remained 19 species belong to an undetermined unit or to more units for the moment. Apparently two species Palicourea locellata and $P$. woronowii do not fit well into Nonatelia, and even to the genus Palicourea either. For the separation of $P$. woronowii there is not sufficient information, but in the case of $P$. locellata the morphological information available are well-enough for its distinction on generic level. The new genus is dedicated to the author of the species, being Tromlyca the anagram of $\mathrm{C}$. M. Taylor.

Key words: new genus, Nonatelia, Palicourea, Tromlyca, Palicoureeae, Rubiaceae

\section{INTRODUCCIÓN}

C. M. Taylor recientemente publicó la revisión taxonómica de la sección Nonatelia (in Taylor y Hollowell, Novon 2016) ampliando el grupo originalmente monotípico hasta 26 especies según un concepto nuevo. Borhidi (2016) en su trabajo monográfico sobre la circumscripción del subgénero Heteropsychotria considera, que la sección en la forma propuesta por Taylor es heterogéneo, un "cajón de sastre" utilizando la terminología de David Lorence (Flora Mesoamericana 4(2): 17. 2012). Solamente 5 especies de 26 corresponde a la descripción original de Nonatelia Aubl. que pueden mantenerse dentro de 
la sección. Otras 19 especies forman tres distintos grupos más o menos bien caracterizables. Finalmente hay dos especies, $P$. locellata y $P$. woronowii, que tienen caracteres obvíamente muy distintos de las demás especies clasificadas en Nonatelia sensu Taylor y no corresponden a los criterios utilizados en el trabajo. Ellas representan probablemente dos géneros desconocidos. Para describirlos cómo nuevos, en el caso de Palicourea woronowii (Standl.) C. M. Taylor, Bruniera et Zappi (Rudgea woronowii Standl.) las informaciones morfológicas existentes son insuficientes para la separación taxonómica de la especie al nivel genérico. Al contrario, en el caso de Palicourea locellata C. M. Taylor las informaciones morfológicas conocidas son suficientes para la caracterización y separación confiable de la espécie al rango genérico, como sigue:

\section{Tromlyca Borhidi, genus novum Rubiacearum (tribu Palicoureeae), hoc loco}

Frutices vel arbores parvae caulibus teretibus, stipulis in tubum elongatum connatis apice truncatis vel plerumque brevissime divaricatis in glandulis linearibus 1-6 aggregatis terminatis, longe persistentibus, posterius lateraliter fissis et demum caducis, foliis ovato-lanceolatis nervis lateralibus apicem versus curvatis et ante vel ad marginem conjunctis, inflorescentiis terminalibus, paniculatis, in axes laterales 3-6 ramificatis, ramis lateralibus plerumque cymoso-divaricatis, floribus sessilibus solitariis vel 1-3-aggregatis, satis longe bracteatis, 4-meris, corolla tubular-infundibuliforme, tubo supra medium annuliformiter hirsuto lobis corollinis obtusis, staminibus 4, filamentis sub fauce insertis, glabris, anteris linearibus e fauce corollae breviter exsertis; stigmata 2, linearia; fructus globosus, pyrenis 2, dorso longitudinaliter late 3-costatis. ventraliter 1-2-locellatis, lobulis marginibus elongatis suffultis.

Typus generis: Tromlyca locellata (C. M. Taylor) Borhidi, comb. nova

Basónimo: Palicourea locellata C. M. Taylor, Novon 25: 92. (2016). - Tipo: Colombia. Cundinamarca, mpio. Ubala vereda Campo Hermoso hacia El Retiro, cuenca del río Guavio, $4^{\circ} 45^{\prime} 49^{\prime \prime} \mathrm{N}, 73^{\circ} 21^{\prime} 45^{\prime \prime} \mathrm{W}, 1820 \mathrm{~m}$. Col.: Fernández, J., Cárdenas, L.-C., Vera, D. y Paez, V. (16420), 3 Junio 1998; holotipo: COL-480439. - Ilustr.: C. M. Taylor, Novon 25: 78. Fig. 3. (2016).

Diagnosis original of C. M. Taylor: "Palicourea locellata C. M. Taylor is distinguished from P. eggersii (Standl.) C. M. Taylor and P. meieri C. M. Taylor by its larger stipules that are fused into a prolonged tube that splits longitudinally, its longer bracts and its pyrenes with one or more air chambers".

Etymology: Tromlyca is the anagram of C. M. Taylor, excellent expert of the family Rubiaceae and author of the type species. 
Arbustos o arbolitos de hasta $4 \mathrm{~m}$ alto; tallo cilíndrico glabro; hojas opuestas, láminas elípticas a lanceoladas u ovadas, de 6 a $18 \mathrm{~cm}$ de largo y 1.7 a 7 $\mathrm{cm}$ de ancho, cuneadas $\mathrm{u}$ otusas a redondeadas en la base, acuminadas en el ápice con una punta de 0.8 a $1.5 \mathrm{~cm}$ de largo, papiráceas cuando secas, glabras en el haz, glabras o a menudo pubérulas en los nervios ptincipales del envés, nervios secundarios 9 a 13 pares, ligeramente encorvados conectados en o ceraca del margen, sin o a veces con 1 a 3 venas intersecundarias o tal vez venas terciárias bien evolutas; sin domácias, nervio medio agudamente prominente en ambas caras venas secundarias prominentes, la venación terciária y algo de las cuatrnárias promínulas; el margen tenue y cartilaginoso; pecíolos de $0.6 \mathrm{a}$ $1.2 \mathrm{~cm}$ de largo, glabros; estípulas deciduas después de la fragmentación de un tubo lanceoloideo, liso de 9 a $18 \mathrm{~mm}$ de largo, soldado alrededor del tallo, glabro, abriendo con 1 (o 2) hendiduras longitudinales provocadas por el tallo engrosado, subtruncado en el ápice, terminando en 6 a 12 glándulas lineares de $2-2.3 \mathrm{~mm}$ de largo arregladas en 2 grupitos persistentes. Inflorescencias terminales, paniculadas, pubérulas, de color desconocido, pero probablemente verdes o blancúzcas; pedúnculo de 1 a $2.5 \mathrm{~cm}$ de largo, la parte ramificada piramidal de 4 a 7 por 3.5 a $6 \mathrm{~cm}$, ejes en 3 a 4 ordenes; los secundarios 3 a 6 pares, opuestos a irregularmente arreglados y subverticilados; bracteas estrechamnete triangulares o estrechamente liguladas, glabrescentes, obtusas o agudas, las de los ejes secundarios de 4 a $9 \mathrm{~mm}$ de largo, las de los ejes de más alto rango, mayormente reducidas, las de las flores de 2 a $5 \mathrm{~mm}$ de largo. Flores sésiles, solitarias y en grupos de 2 a 3 en los ejes dicasiales, cada una subtendida por 1 o 2 brácteas lineares, hipantio elipsoidal a subgloboso, ca. $0.8 \mathrm{~mm}$ de diámetro, densamente pubérulo; limbo del cáliz de 0.2 a $0.5 \mathrm{~mm}$ de largo, pubérulo, lóbulos 4 o 5 irregular, triangulares, obtusos o agudos o a veces acuminados; corola tubular-embudada, blanca o de color crema pubérula por fuera; tubo de hasta $7 \mathrm{~mm}$ de largo en botón derecho en la base o muy poco abollada, 1 a $1.2 \mathrm{~mm}$ de ancho en la mitad, glabra por dentro excepto del anillo hirsuto de ca. $2.5 \mathrm{~mm}$ de ancho en la mitad; lóbulos 4, ligulados hasta 3 $\mathrm{mm}$ de largo, lisos por fuera, obtusos; anteras 4, de ca. $2 \mathrm{~mm}$ de largo, con filamentos de ca. $4 \mathrm{~mm}$ de largo e insertado cerca del ápice del tubo de la corola; estigmas 2, lineares, de ca. $2 \mathrm{~mm}$ de largo, situados justamente bajo el tubo de la corola. Infrutescencias similares a las inflorescencias. Fruto subgloboso a deprimido globoso de ca. 4 por $4.5 \mathrm{~mm}$, glabro, color no anotado; pirenos 2, generalmente hemisféricos, con ca. 3 crestas longitudinales anchamente angulosas en el dorso, y ventralmente con 1 o 2 cámeras redondeadas, con apéndices alargadas longitudinales en el margen. 


\section{DISCUSIÓN}

Al estudiar cuidadosamente la descripción y caracterización de la sección Nonatelia (Aubl.) C. M. Taylor en su tratamiento taxonómico (Novon 25: 80. 2016) aparece, que no hay un carácter morfológico común válido para todas las especies clasificadas en la sección. Pués, el grupo taxonómico es morfológicamente heterogéneo.

El uso del término Nonatelia Aubl. en el tratamiento de concepto ampliado de C. M. Taylor pierde su sentido original completamente. Porque el carácter decisivo de Nonatelia Aubl. es, que el número de los estigmas, de los lóculos del ovario, de los lóbulos del disco y de los pirenos es 5 , son cuneiformes, los lóbulos de la corola terminan en apéndices o cuernos de 0.2 a $1.2 \mathrm{~mm}$ de largo. Yo prefiero mantener la sección Nonatelia en el sentido y emendación siguiente.

Sect. Nonatelia (Aubl.) C. M. Taylor emend. Borhidi, hoc loco

Inflorescentia paniculata elongata et angustata, racemiformis, flores cum lobis corollinis breviter appendiculatis, stylis 5-stigmatibus, disco 5-lobato, bacca 5-partita, pyrenis 5, basi cuneatis suffulti.

Typus sectionis: Palicourea racemosa (Aubl.) Borhidi

Species adhuc pertinentes: Palicourea dodsoniana C. M. Taylor, Palicourea racemosa (Aubl.) Borhidi, Palicourea stenostachya (Standl.) C. M. Taylor, Palicourea subfusca (Müll. Arg.) C. M. Taylor, Palicourea tsakiana (C. M. Taylor) C. M. Taylor.

Las demás 19 species clasificadas en Nonatelia por C. M. Taylor tienen una posición taxonómica dudosa, posiblemente pertenecientes en varios grupos de especies.

Para la separación morfológica del género nuevo Tromlyca se encuentran datos ofrecidos claramente en el protólogo de la sección Nonatelia, presentados por el mismo autor, C. M. Taylor, que son los siguentes:

1) Las mayoría de las especies tiene tallos cuadrangulares o ligeramente aplanados, mientras Palicourea locellata tiene tallos cilíndricos, además ligeramente ensanchados o inflados sobre los nodos superiores, según el dibujo presentado por C. M. Taylor (Novon 25: 78, Fig. 3B).

2) Las estípulas son persistentes, soldadas alrededor del tallo, con 2 lóbulos en el lado interpeciolar, estos son estrechamente triangulares, lineares o liguladas a veces fusionadas en un lobo, o a menudo son bífidos a multífidos, terminando en glándulas, mientras Palicourea locellata tiene estípulas comple- 
tamente distintas, fusionadas en una vaína larga, abriendose con 1 o 2 lateral hendiduras longitudinales y caedizas, lóbulos apicales reducidas terminando en dos grupos de glándulas persistentes formados por glándulas lineares 0 cilíndricas. La apertura de la vaína es generalmente espatiforme. (Novon 25: 78, Fig. 3C).

3) Las flores de la mayoría de las especies de la sección Nonatelia son 5-meras, mientras la Palicourea locellata tiene flores 4-meras y lóbulos de la corola redondeados a obtusos. La especie considerderada como próxima $P$. eggersii tiene flores 4-meras y 5-meras también (Steyermark: Flora de Venezuela 9: 1330. Fig. 208) lóbulos de la corola apiculados.

4) La mayoría de las especies de la sección Nonatelia tienen bracteas y bractéolas aovadas a lanceoladas cortas, de 1-3 mm de largo, $P$. soejartoi tiene bráceas triangulares de 2-6 $\mathrm{mm}$ de largo, mientras las bracteas de $P$. locellata son linear-lanceoladas y 4-9 mm de largo (Novon 25: 78, Figs 3A y D).

5) Las especies de la sección Nonatelia todas tienen pirenos hemisféricos, convexos en la cara dorsal con 3-5 crestas longitudinales, planas en la cara ventral con margenes obtusos, no apendiculados. En contraste con las demás especies de la sección $P$. locellata tiene pirenos especiales, porque la porción ventral del pireno tiene 1 o 2 cámeras vacías un carácter desconocido en el género Palicourea, además el márgen del pireno esta alargada y termina en un apéndice o ala que se extiende en dirección adaxial (Novon 25: 78, Fig. 3E).

Considero, que las caracteristicas disyuntivas aqui mencionadas son importantes, sobre todo el típo de las estípulas y del fruto que son completamente ajenos y desconocidos en el género Palicourea. Quiero acentuar, que la morfología de las estípulas y de los pirenos está ampliamente aceptada y reconocida como elementos decisivos en la caracterización y delimitación de los géneros en el complejo de Psychotrieae-Palicoureeae.

\section{LITERATURA CONSULTADA}

Andersson, L. (2001): Margaritopsis (Rubiaceae, Psychotrieae) is a pantropical genus. Syst. Geogr. Pl. 71: 73-85. https://doi.org/10.2307/3668754

Andersson, L. (2002a): Relationships and generic circumscriptions in the Psychotria complex (Rubiaceae, Psychotrieae). - Syst. Geogr. Pl. 72: 167-202. https://doi.org/ $10.2307 / 3668767$

Andersson, L. (2002b): Re-establishment of Carapichea (Rubiaceae, Psychotrieae). - Kew Bull. 57: 363-374. https://doi.org/10.2307/4111112

Andersson, L. y Rova, J. H. E. (1999): The rps16 intron and the phylogeny of the Rubiaceae. - Plant Syst. Evol. 214: 161-186. https://doi.org/10.1007/BF00985737

Borhidi, A. (2011): Transfer of the Mexican species of Psychotria subgen. Heteropsychotria to Palicourea based on morphological and molecular evidences. - Acta Bot. Hung. 53: 241-250. https://doi.org/10.1556/ABot.53.2011.3-4.4 
Borhidi, A. (2012): Rubiáceas de México. Segunda y ampliada edición. - Akadémiai Kiadó, Budapest, $610 \mathrm{pp}$.

Borhidi, A. y Oviedo-Prieto, R. (2015): Correcciones a la taxonomía y nomenclatura del género Psychotria s. 1. en las floras de Cuba, La Española y Jamaica. - Acta Bot. Hung. 57: 271-278. https://doi.org/10.1556/034.57.2015.3-4.2

Borhidi, A., Martínez-Salas, E. y Ramos Álvarez, C. H. (2015): An uncommon pyrene type and two new genera in the neotropical Psychotrieae (Rubiaceae). - Acta Bot. Hung. 57: 241-270. https://doi.org/10.1556/034.57.2015.3-4.1

Borhidi, A. L., Oviedo-Prieto, R. y Fernández-Zequeira, M. (2016): Nuevos resultados de la revisión taxonómica de los géneros Palicourea y Psychotria (Rubiaceae, Psychotrieae) en Cuba. - Acta Bot. Hung. 58: 1-48. https://doi.org/10.1556/034.58.2016.1-2.1

Bremer, B. (2009): A review of molecular phylogenetic studies of Rubiaceae. - Ann. Mo. Bot. Gard. 96: 4-26. https://doi.org/10.3417/2006197

Burger, W. C. y Taylor, C. M. (1993): Family \# 202 Rubiaceae. In: Burger, W. C. (ed.): Flora Costaricensis. - Fieldiana, Bot., n.s., 33: 1-333.

Dwyer, D. J. (1980a): Rubiaceae. In: Woodson, R. E. Jr., Scheryl, R. W., et al. (eds): Flora of Panama. Part 1. - Ann. Mo. Bot. Gard. 67(1): 1-256. https://doi.org/10.2307/2398954

Dwyer, D. J. (1980b): Rubiaceae. In: Woodson, R. E. Jr., Scheryl, R. W., et al. (eds): Flora of Panama. Part IX. Family 179. Rubiaceae. Part II. - Ann. Mo. Bot. Gard. 67(2): 257-522. https://doi.org/10.2307/2398901

Govaerts, R. (2016): World checklist of Rubiaceae. - Royal Botanic Gardens, Kew. http://apps. kew.org/wcsp

Nepokroeff, M., Bremer, B. y Sytsma, K. J. (1999): Reorganization of the genus Psychotria and tribe Psychotrieae (Rubiaceae) inferred from ITS and rbcL sequence data. - Syst. Bot. 24(1): 5-27. https://doi.org/10.2307/2419383

Petit, E. (1964): Les espèces africaines du genre Psychotria L. Rubiaceae. I. - Bull. Rijksplantentuin (Brussel) 34: 1-228. https://doi.org/10.2307/3667213

Piesschaert, F., Robbrecht, E. y Smets, E. (2001): Patterns in pyrenes: the systematic significance of pyrene morphology in Chassalia (Rubiaceae, Psychotrieae) and related genera. - Flora 196: 121-131. https://doi.org/10.1016/s0367-2530(17)30027-0

Razafimandimbison, S. G., Taylor, C. M., Wikström, N., Pailler, T., Khodabandeh, A. y Bremer, B. (2014): Phylogeny and generic limits in the sister tribes Psychotrieae and Palicoureeae (Rubiaceae): evolution of schizocarps in Psychotria and origin of bacterial leaf-nodules of the Malgasy species. - Amer. J. Bot. 101(7): 1102-1126. http:// dx.doi.org/10.3732/ajb.1400076

Robbrecht, E. (1988): Tropical woody Rubiaceae. Characteristic features and progressions. Contributions to a new subfamilial classification. - Opera Bot. Belg. 1: 1-271.

Robbrecht, E. (1989a): Germination slits in Rubiaceae diaspores. - Progr. y Abstr., Flora Males. Symp., Leiden, August, 1989, p. 53.

Robbrecht, E. (1989b): A remarkable new Chazaliella (African Psychotrieae), exemplifying the taxonomic value of pyrene characters in the Rubiaceae. - Bull. Mus. natn. Hist. Nat. Paris, sér. 4, 11: 341-345.

Schnell, R. (1960): Note sur le genre Cephaëlis et le problème de l'évolution parallèle chez les Rubiacées. - Bull. Rijksplantentuin (Brussel) 30: 357-373. https://doi.org/ $10.2307 / 3667307$

Standley, P. C. (1918-1934): Rubiaceae. - North American Flora 32: 1-300.

Standley, P. C. (1930): Rubiaceae of Colombia. - Publ. Field Mus. Nat. Hist., Bot. Ser. 7(1): $1-175$. 
Standley, P. C. (1938): Rubiaceae of Venezuela. - Publ. Field Mus. Nat. Hist., Bot. Ser. 7(4): 343-485.

Steyermark, J. A. (1972): Rubiaceae. In: Steyermark, J. A., Maguire, B., Wurdack, J. J. et al. (eds): Botany of the Guyana Highland, Part IX. - Mém. New York Bot. Gard. 23: 227-832.

Steyermark, J. A. (1974): Rubiaceae. - In: Lasser, T. y Steyermark, J. A. (eds): Flora de Venezuela. Inst. Bot., Caracas, Vol. 9(1-3), pp. 1-2070.

Taylor, C. M. (1989): Revision of Palicourea in Mexico and Central America. - Syst. Bot. Monogr. 26: 1-102. https://doi.org/10.2307/25027720

Taylor, C. M. (2001): Overview of the neotropical genus Notopleura (Rubiaceae, Psychotrieae) with the description of some new species. - Ann. Mo. Bot. Gard. 88: 478-515. https://doi.org/10.2307/3298587

Taylor, C. M. (2004): Rubiacearum Americanarum Magna Hama Pars XVI. New species, a new subspecies, and an overlooked species of Psychotria subg. Heteropsychotria from Mexico, Central America, and western South America. - Novon 14(4): 493-508.

Taylor, C. M. (2005): Margaritopsis (Rubiaceae, Psychotrieae) in the Neotropics. - Syst. Geogr. Pl. 75(2): 161-177. https://doi.org/10.2307/3668574

Taylor, C. M. (2012a): Palicourea Aubl. - In: Davidse, G., Sousa, M., Knapp, S. y Chiang, F. (eds): Flora Mesoamericana, Vol. 4/2, pp. 163-181.

Taylor, C. M. (2012b): Psychotria L. - In: Davidse, G., Sousa, M., Knapp, S. y Chiang, F. (eds): Flora Mesoamericana, Vol. 4/2, pp. 192-241.

Taylor, C. M. (2015a): Rubiacearum Americanarum Magna Hama Pars XXXIII. The new group Palicoureae sect. Didymocarpae with four new species and two new subspecies (Palicoureeae). - Novon 23(4): 452-478. https://doi.org/10.3417/2012003

Taylor, C. M. (2015b): Rubiacearum Americanarum Magna Hama Pars XXXIV. The new group Palicourea sect. Tricephalium with eight new species and a new subspecies (Palicoureeae). - Novon 24(1) 55-95. https://doi.org/10.3417/2015001

Taylor, C. M. y Gereau, R. E. (2013): The genus Carapichea (Rubiaceae, Psychotrieae). Ann. Mo. Bot. Gard. 99: 100-127. https://doi.org/10.3417/2011064

Taylor, C. M. y Hollowell, V. C. (2016): Rubiacearum Americanarum Magna Hama Pars XXXV. The new group Palicourea sect. Nonatelia with five new species (Palicoureeae). - Novon 25: 69-110. https://doi.org/10.3417/2015012

Taylor, C. M. y Lorence, D. H. (1992): Notes on Psychotria subgenus Heteropsychotria (Rubiaceae: Psychotrieae) in Mexico and Northern Central America. - Novon 2: 259-266. https://doi.org/10.2307/3391564

Taylor, C. M., Steyermark, J. A., Delprete, P., Persson, C., Costa, C., Vicentini, A. y Cortés, R. (2004): Rubiaceae. - In: Berry, P., Yatskievych, K. y Holst, B. K. (eds): Flora of the Venezuelan Guayana. Vol. 8. Missouri Bot. Gard. Press, St. Louis, pp. 497-847.

Verdcourt, B. (1976): Rubiaceae (part 1). - In: Polhill, R. M. (ed.): Flora of Tropical East Africa. Agents for Overseas Governments and Administration, London, 414 pp.

WCSP (2014): World checklist of selected plant families. - Royal Botanic Gardens, Kew, http:// apps.kew.org./wcsp/, (accessed 6. December 2014). 